\title{
MR Imaging Scoring System for White Matter Injury after Deep Medullary Vein Thrombosis and Infarction in Neonates
}

\author{
(D) K.L. Benninger, (D) N.L. Maitre, DL. Ruess, and (D).A. Rusin
}

\begin{abstract}
BACKGROUND AND PURPOSE: Advanced imaging techniques have allowed earlier and more accurate detection of cerebral deep medullary vein thrombosis and infarction. Our objective was to develop an MR imaging scoring system to evaluate the severity of white matter injury in neonates with deep medullary vein thrombosis and infarction.
\end{abstract}

MATERIALS AND METHODS: This was a retrospective study of infants born $\geq 32$ weeks' gestation (2000-2016) diagnosed with deep medullary vein thrombosis and infarction on neuroimaging in the first 30 days of life. A 102-point deep medullary vein white matter injury global severity score was developed. MR images were scored by 2 pediatric radiologists. Subject clinical data and regional and global severity scores were recorded.

RESULTS: Fifty-one patients (mean gestational age, $37.3 \pm 2.2$ weeks; mean birth weight, $3182 \pm 720 \mathrm{~g}$ ) were included with a mean age at diagnosis via MR imaging of postnatal day $10.1 \pm 6.1$. Global severity scores ranged from 1 to 53 , with a median score of 11 (interquartile range, 5-25). Lesions were more common in the frontal and parietal regions and less common in the occipital and temporal regions. Fifty-five percent of the group had neonatal seizures. No difference in perinatal risk factors (gestational age, birthweight, 5-minute Apgar score, chorioamnionitis, delivery room resuscitation, ventilator, or inotrope requirement) was observed among severity score quartiles.

CONCLUSIONS: An MR imaging scoring system provides a comprehensive and objective classification of WM injury after deep medullary vein thrombosis and infarction in late preterm and term neonates. The global severity score is independent of gestational age and other antenatal risk factors, consistent with presentation in previously healthy-appearing neonates.

ABBREVIATION: DMV = deep medullary vein

erebral sinovenous thrombosis is an important cause of morbidity and mortality in children, with an incidence of 0.67 cases per 100,000 children, with nearly half of cases occurring in neonates. ${ }^{1,2}$ Neonates are at increased risk of developing throm-

\footnotetext{
Received September 26, 2018; accepted after revision December 1.

From the Departments of Pediatrics and Center for Perinatal Research (K.L.B., N.L.M.) and Radiology (L.R., J.A.R.), Nationwide Children's Hospital, Columbus, Ohio; and Wexner Medical Center (K.L.B., N.L.M., L.R., J.A.R.), Ohio State University, Columbus, Ohio.

This work was supported by 1R01HD081120-01A1 from the Eunice Kennedy Shriver National Institute of Child Health and Human Development to N.L.M.

The content is solely the responsibility of the authors and does not necessarily represent the official view of the funding organization.

Paper previously presented at: Annual Meeting of the Midwest Society for Pediat ric Research, October 4-5, 2018, Royal Oak, Michigan; and Children's Hospitals Neonatal Consortium 10th Annual Quality \& Research Symposium, October 22-24, 2018, Lewis Center, Ohio.

Please address correspondence to Kristen L. Benninger, MD, Nationwide Children's Hospital, 575 Children's Crossroad, WB5203, Columbus, OH 43215-5994; e-mail: kristen.benninger@nationwidechildrens.org

- Indicates open access to non-subscribers at www.ajnr.org

http://dx.doi.org/10.3174/ajnr.A5940
}

bosis secondary to immature hemostatic systems. ${ }^{3,4}$ Other recognized predisposing risks include maternal and perinatal factors unique to the neonatal time period such as maternal preeclampsia, diabetes and chorioamnionitis, fetal distress, birth asphyxia, sepsis, cardiac defects, and inherited prothrombotic abnormalities. ${ }^{1-3}$

The incidence of cerebral sinovenous thrombosis may be underestimated in neonates. Cranial sonography is still the most routinely used neuroimaging technique in this age group, yet it is insufficient to precisely identify deep or diffuse thromboses. However, central nervous system imaging using MR imaging and, in particular, SWI allows accurate localization of deep vein thrombosis or infarction in children and adults. ${ }^{5-7}$ In particular, linear, radially oriented, fan-shaped lesions in the periventricular WM are indicative of deep medullary vein (DMV) involvement. This particular imaging pattern is explained by the anatomic distribution of the WM venous drainage. ${ }^{8}$ The characteristic pattern of DMV thrombosis and ensuing infarction of the surrounding tissue has been recognized on neuroimaging with increasing frequency in the past decade. , $6,9^{-1}$

AJNR Am J Neuroradiol 40:347-52 Feb 2019 www.ajnr.org 


\begin{tabular}{|c|c|c|}
\hline & Points & Scoring \\
\hline Cerebral edema & $0-3$ & None $=0$, right or left $=1$, bilateral asymmetric $=2$, bilateral symmetric $=3$ \\
\hline Corpus callosum & $0-3$ & 1 point each for genu, body, and/or splenium lesion \\
\hline $\begin{array}{l}\text { Right cerebral WM } \\
\text { Frontal } \\
\text { Parietal } \\
\text { Occipital } \\
\text { Temporal }\end{array}$ & $\begin{array}{l}0-12 \\
0-12 \\
0-12 \\
0-12\end{array}$ & $\begin{array}{l}\text { Score each cerebral hemisphere region for punctate, linear, and/or cavitary lesions } \\
\text { Punctate: mild }(1-3 \text { lesions })=1 \text {, moderate }(4-6 \text { lesions })=2 \text {, severe }(>6 \text { lesions })=3 \\
\text { Linear: mild }(1-3 \text { lesions })=1 \text {, moderate }(4-6 \text { lesions })=2 \text {, severe }(>6 \text { lesions })=3 \\
\text { Cavitary: } \text { mild }(1-3 \text { lesions, }<15 \mathrm{~mm})=2 \text {, moderate }(4-6 \text { lesions, }<15 \mathrm{~mm})=4 \\
\text { severe }(>6 \text { lesions, }<15 \mathrm{~mm} \text {, or } 1+\text { lesion }>15 \mathrm{~mm})=6\end{array}$ \\
\hline $\begin{array}{l}\text { Left cerebral WM } \\
\text { Frontal } \\
\text { Parietal } \\
\text { Occipital } \\
\text { Temporal }\end{array}$ & $\begin{array}{l}0-12 \\
0-12 \\
0-12 \\
0-12\end{array}$ & \\
\hline & $0-102$ & Global severity score \\
\hline
\end{tabular}

Despite increasing recognition of DMV thrombosis and infarction on neuroimaging, there is no published standardized approach to evaluate or grade these lesions in neonates. Clinical outcome data related to DMV pathology in neonates or children exist only in isolated case reports of motor impairment. ${ }^{9}$ Fetal diagnosis of DMV thrombosis and infarction has been described; however, in all cases in utero fetal or neonatal death shortly after delivery occurred. ${ }^{10,11}$ Similar lesions are described more frequently in preterm neonates with germinal matrix hemorrhage and congestion and obstruction of the terminal or medullary veins with subsequent parenchymal hemorrhage. ${ }^{5}$ However, in term neonates, germinal matrix hemorrhage is much less frequent, but DMV congestion, thrombosis, infarction, and hemorrhage are also seen. ${ }^{5}$ It has been hypothesized that DMV lesions in term neonates may be related to transient hypoperfusion or impairment in cerebral blood flow. ${ }^{5}$ No current system classifies this problem in infants during the perinatal period.

Therefore, the aim of our study was to develop a scoring system for conventional MR imaging that comprehensively and objectively defines brain injury after DMV thrombosis and infarction, to facilitate future prospective studies of developmental outcomes. We hypothesized that we could develop a semiquantitative system to characterize both the extent and topography of insults to the neonatal brain after DMV thrombosis and infarction. To test this hypothesis, we leveraged a large retrospective neuroimaging data base at our institution and graded DMV lesions while characterizing perinatal risk factors consistent with cerebral sinovenous thrombosis.

\section{MATERIALS AND METHODS Study Design and Participants}

We retrospectively reviewed patients with a diagnosis of DMV thrombosis and infarction in a pediatric neuroradiology data base. All subjects were admitted to the neonatal intensive care unit or other hospital units at our institution between 2000 and 2016. Included in the review were infants born at $\geq 32$ weeks' gestation and diagnosed with DMV thrombosis and infarction on neuroimaging during the first 30 days after birth. We excluded infants with congenital CNS anomalies or major congenital syndromes. The electronic medical record was reviewed, and all pertinent clinical, laboratory, imaging, and follow-up data were collected. This study was approved by the institutional (Nationwide Children's Hospital) review board for human research of the hospital.
Study data were managed using REDCap electronic data capture tools (https://www.project-redcap.org/) hosted at our institution. $^{12}$

\section{MR Imaging Acquisition}

All brain MR imaging scans were obtained on either 1.5T or 3T MR imaging scanners (Excite HDXT 1.5T and GE Signa MR750 3T; GE Healthcare, Milwaukee, Wisconsin; and Magnetom Espree 1.5T and Magnetom Skyra 3T; Siemens, Erlangen Germany). Due to the retrospective study design, there was variation in imaging protocols, sequences, and parameters obtained. Scans included a combination of T1, T2, T1 FLAIR, DWI, and SWI sequences. Twenty patients also had 2D time-of-flight MR venograms available for review.

\section{MR Imaging Assessment}

While all scans for all subjects were reviewed, the MR image obtained at the time of initial radiologic diagnosis of DMV thrombosis and infarction was used for scoring purposes. A standardized scoring system was developed a priori on the basis of the topography of venous supply to the $\mathrm{WM},{ }^{8}$ to describe the severity of cerebral WM injury after DMV thrombosis and infarction.

The DMV WM injury-severity scoring system identified and assigned points for the degree of injury for focal lesions within the right and left frontal, parietal, occipital, and temporal lobes, for focal infarction within the corpus callosum and for diffuse WM cerebral edema (Table 1). Focal infarction and diffuse cerebral swelling were identified as focal or diffuse WM increased T2 and/or restricted diffusion signal abnormality, respectively, and were scored as present in cerebral WM either unilaterally, bilateral symmetrically, or bilateral asymmetrically. Regions were then scored separately for any punctate, linear, and/or cavitary lesions. Punctate and linear lesions, including those in a radial pattern, were identified as T1 bright and T2 dark, with hemorrhage confirmed on SWI and scored as mild, moderate, or severe on the basis of the number of lesions. Cavitary lesions were more heavily weighted to account for their larger and more severe tissue injury. All regional scores were added to the corpus callosum and cerebral edema scores to yield a global severity score ( $0-102$ points). A diagrammatic representation of the scoring system is shown in Figs 1-4. Two pediatric radiologists independently scored the 
scans and then agreed on the final consensus scoring after review of any discrepancies.

\section{Statistical Analysis}

Continuous variables were summarized using mean $\pm \mathrm{SD}$ or median (interquartile range). The DMV WM injury-severity scoring

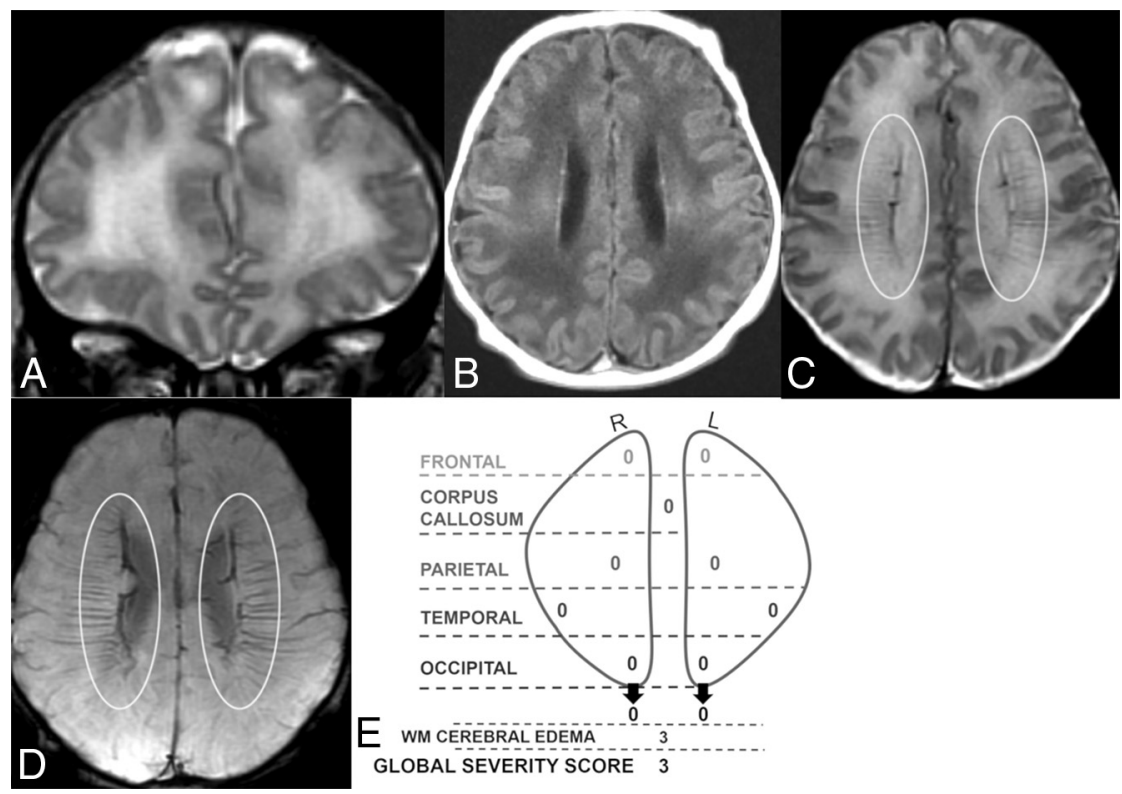

FIG 1. Coronal T2 (A) and axial T1 FLAIR (B), T2 (C), and SWI (D) MR images of a 6-day-old boy. Global severity score $=3$ (first quartile) (E). There is diffuse bilateral WM signal abnormality of cerebral edema without infarction (3 points) $(A$ and $B$ ). The DMVs are prominent bilaterally, seen on T2 (C) and SWI (D) (ovals). No WM lesions are visible. R indicates right; L, left. system is a sum of scaled scores with a continuous composite. Categoric variables were summarized using percentiles. ANOVA was used to compare variance in means among the 4 quartiles in continuous variables. Adjustment for multiple comparisons was through Bonferroni correction. The Kruskal-Wallis test for nonparametric variables was used to assess the frequency distribution of categoric variables. Significance was assigned at $P<.05$. All analyses were performed using SPSS Statistics for MacIntosh (Version 24.0; Release 2016; IBM, Armonk, New York).

\section{RESULTS}

Our cohort consisted of 51 patients. Infants were born at a mean gestational age of 37 weeks (range, 32-41 weeks) with a mean birth weight of $3182 \pm 720 \mathrm{~g}$. Sixty-three percent of the group was male. On average, infants were admitted to our hospital on day of life $4.7 \pm 4.9$, with only $37 \%$ admitted within the first day of life. The most commonly identified maternal risk factors were preeclampsia or hypertension (33\%), diabetes $(12 \%)$, and a maternal prothrombotic disorder (6\%). Fifty-five percent of the infants had no identified maternal risk factors. One-third of in-

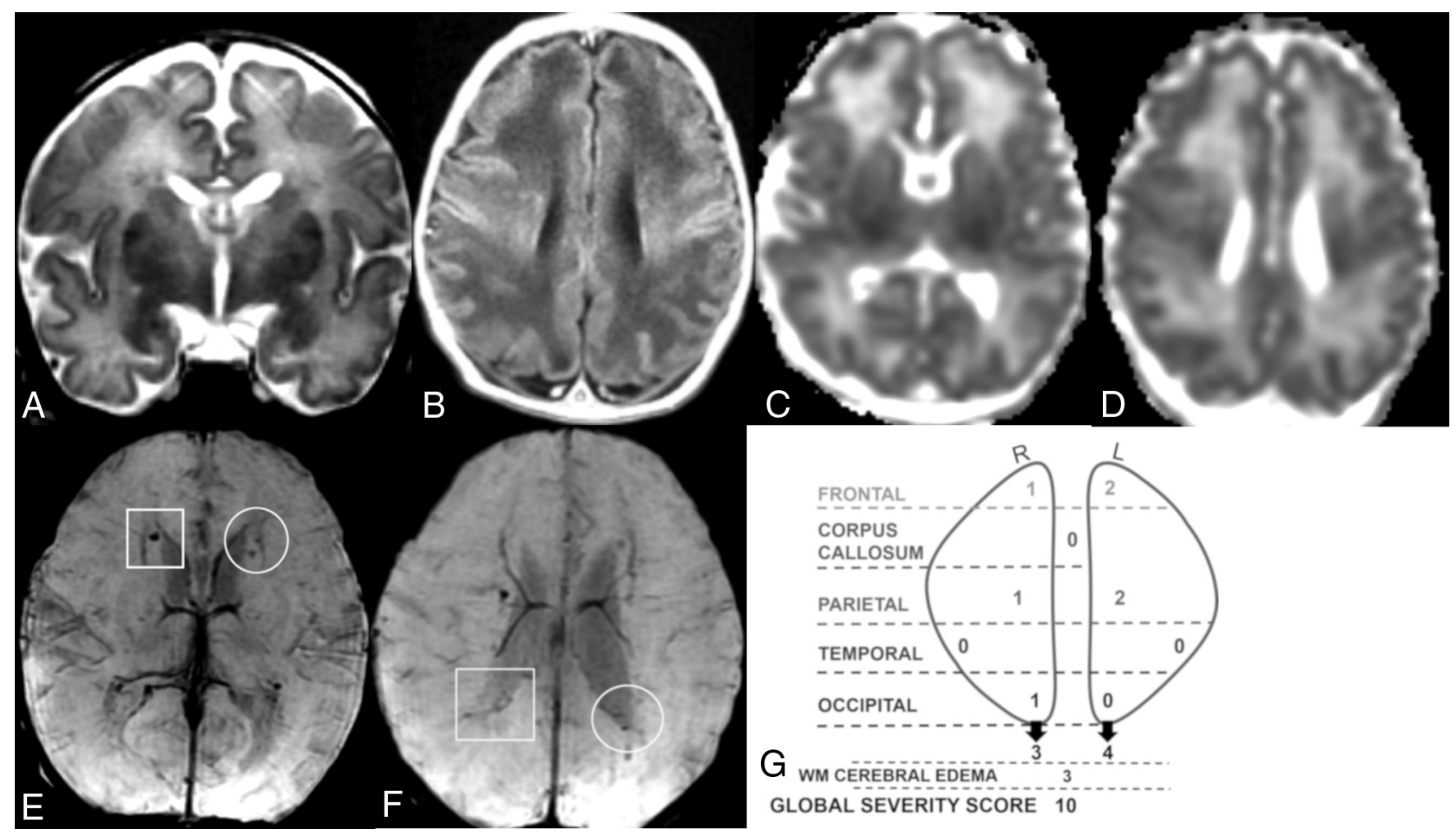

FIG 2. Coronal T2 $(A)$, axial T1 (B), $A D C(C$ and $D)$, and SWI $(E$ and $F)$ MR images of a 7-day-old girl. Global severity score $=10$ (second quartile) $(G)$. There is diffuse bilateral WM cerebral edema (3 points) $(A$ and $B)$ and multiple acute bilateral, asymmetric (left $>$ right) hemorrhagic WM lesions (T1 bright and T2 dark) without restricted diffusion (C-F). On the right, there are mild punctate lesions in the frontal (1 point), parietal (1 point), and occipital WM (1 point, not shown) ( $E$ and $F$, boxes), and on the left, there are moderate punctate lesions in the frontal ( 2 points) and parietal WM (2 points) ( $E$ and $F$, circles). No corpus callosum or temporal region WM lesions are visible. R indicates right; L, left. 
fants demonstrated signs of fetal distress during the labor and delivery process (decelerations or a nonreassuring biophysical profile). Twenty percent were intubated, and $4 \%$ required chest compressions or epinephrine during the postdelivery resuscita-

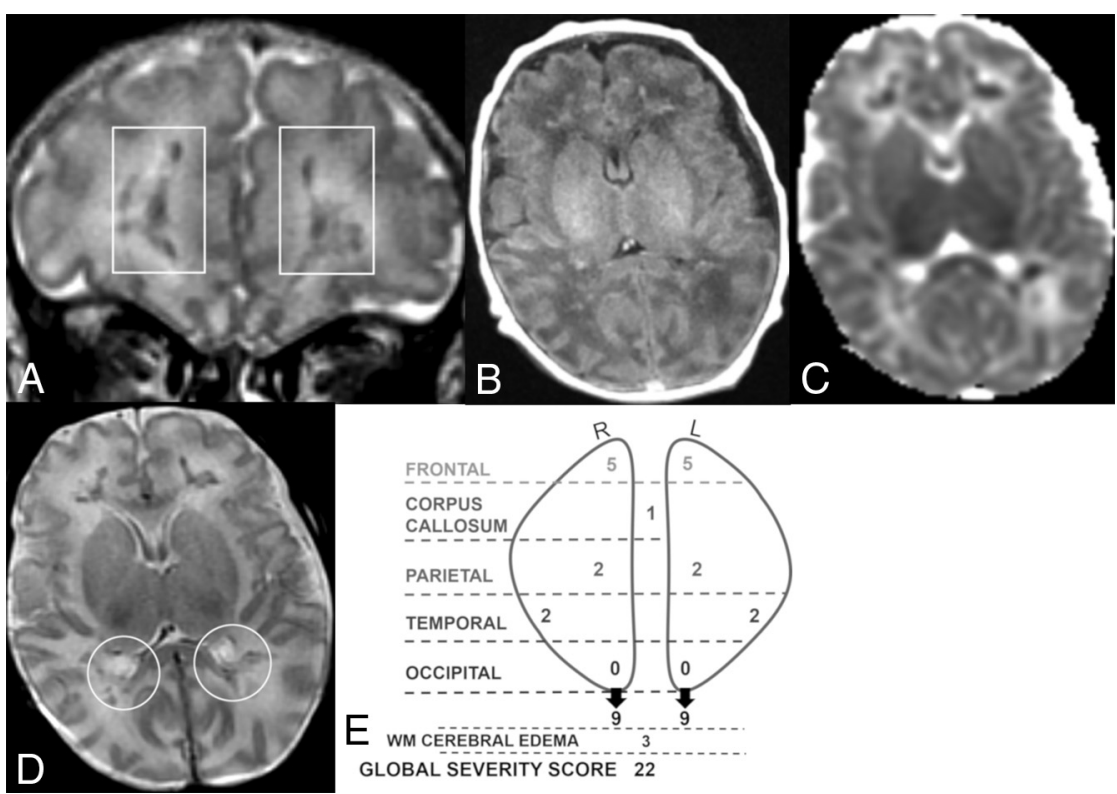

FIG 3. Coronal T2 (A) and axial TI FLAIR (B), ADC (C), and T2 (D) MR images of a 21-day-old boy. Global severity score $=22$ (third quartile) $(E)$. There is diffuse bilateral WM cerebral edema (3 points) $(A-D)$ and multiple acute bilateral symmetric hemorrhagic WM lesions (T1 bright and T2 dark): frontal (moderate punctate, 2 points, and severe linear, 3 points) (A, boxes), parietal (moderate punctate, 2 points) $(D$, circles), and temporal regions (moderate punctate, 2 points, not shown). There are no visible occipital WM lesions. R indicates right; L, left. tion. The average 5-minute Apgar score was $7.8 \pm 1.7$. Neonatal comorbidities included encephalopathy $(20 \%)$, neonatal infection (18\%), hypoglycemia (35\%), complex congenital heart disease $(8 \%)$, and prothrombotic disorders $(8 \%)$. Forty-three percent of infants required mechanical ventilation, and $18 \%$ required inotropes in the first week of life. Infants presented on day of life $4.5 \pm 5.3$ with seizures (55\%), apnea (53\%), lethargy (49\%), and poor feeding (35\%). The average age at referral of these infants to our institution was $4.7 \pm 4.8$ days, and the average age at diagnosis via MR imaging was postnatal day $10.1 \pm 6.1$.

The DMV WM injury global severity scores ranged from 1 to 53 and were divided into quartiles. The median score for the cohort was 11 (interquartile range [25th-75th percentile], 5-25). Seventy-six percent and $73 \%$ of patients had lesions in the frontal and parietal WM, respectively. Twelve percent and $41 \%$ of patients had lesions in the occipital and temporal WM, respectively. Acute parenchymal and corpus callosum lesions showed restricted diffusion. Representative MR images from a patient in each quartile with corresponding diagrammatic scoring are shown in

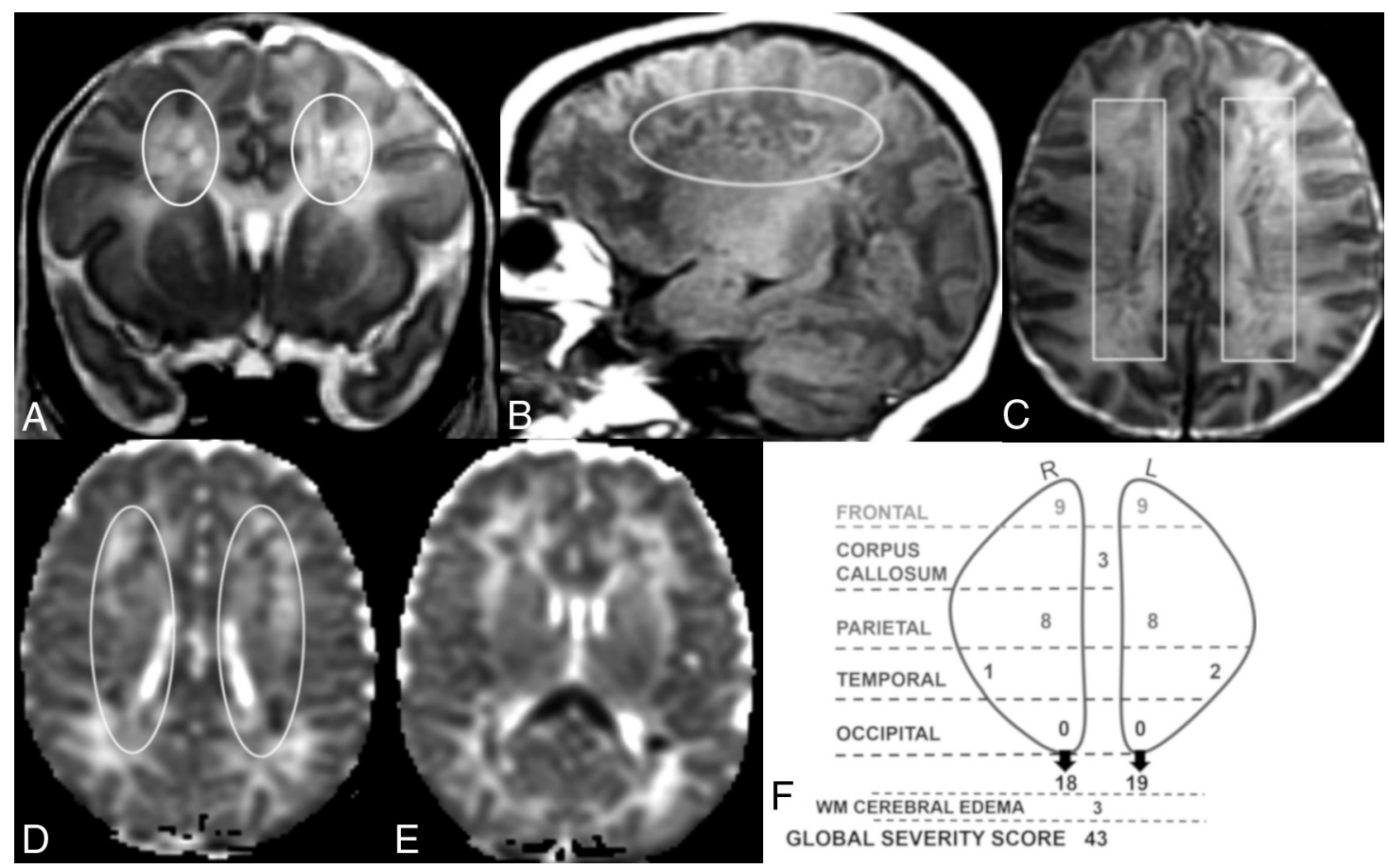

FIG 4. Coronal $T 2(A)$ and sagittal $T 1(B)$, axial $T 2(C)$, and axial $A D C(D$ and $E)$ MR images of a 12 -day-old boy. Global severity score $=43$ (fourth quartile) (F). There is diffuse bilateral WM cerebral edema (3 points) and multiple bilateral hemorrhagic WM lesions with restricted diffusion $(A-F)$. There are symmetric bilateral frontal and parietal lesions: severe linear lesions ( 3 points; $C$, boxes) and severe cavitary lesions with restricted diffusion ( 6 points; $A, B$, and $D$, ovals), There are bilateral temporal lesions: right (mild punctate, 1 point) and left (moderate punctate, 2 points, not shown). There is restricted diffusion throughout the corpus callosum ( 3 points, E). No occipital WM lesions are visible. R indicates right; L, left. 
Figs 1-4, illustrating the range of severity and morphology of the various lesions. Tables 2 and 3 demonstrate the distribution of injury-severity scores by region. Only occipital regional severity scores and WM cerebral edema scores were not significantly different among quartiles.

Perinatal clinical variables were compared with global severity score quartiles (Table 4). Perinatal variables included factors associated with an increased risk of perinatal morbidity and mortality such as the 5-minute Apgar score, chorioamnionitis, small for gestational age, neonatal infection, ventilator requirement, inotrope use, and seizures. We observed no significant difference in perinatal risk factors among the severity score quartiles.

\section{DISCUSSION}

By leveraging the largest cohort to date with imaging for DMV thrombosis and infarction, we developed a neuroimaging scoring system, allowing a comprehensive and objective classification of WM injury in term and late preterm neonates. In our cohort of infants with DMV thrombosis and infarction, WM injury global severity scores were independent of gestational age at birth and other perinatal risk factors for morbidity and mortality. While the average age at referral of these infants to our institution was 4.7 days, the average age at MR imaging diagnosis was 10.1 days, suggesting that this entity is either under-recognized or that patients are not referred for immediate imaging. These factors may

Table 2: Injury severity scores: WM cerebral edema and corpus callosum ${ }^{\mathrm{a}}$

\begin{tabular}{lccccc}
\hline \multicolumn{1}{c}{ Region } & Score 0 & Score 1 & Score 2 & Score 3 & $\boldsymbol{P}$ Value \\
\hline WM cerebral edema & $4(8)$ & $2(4)$ & $9(18)$ & $36(71)$ & .52 \\
Corpus callosum & $26(51)$ & $10(20)$ & $8(16)$ & $7(14)$ & $<.001$ \\
\hline
\end{tabular}

a Data are number of patients (\%).

Table 3: Injury severity scores by region ${ }^{\mathrm{a}}$

\begin{tabular}{lccccc}
\hline \multicolumn{1}{c}{ Region } & Score 0-2 & Score 3-5 & Score 6-8 & Score $\geq 9$ & $\boldsymbol{P}$ Value \\
\hline Right frontal & $24(47)$ & $14(28)$ & $8(16)$ & $5(10)$ & $<.001$ \\
Right parietal & $33(65)$ & $8(16)$ & $9(18)$ & $1(2)$ & $<.001$ \\
Right occipital & $50(98)$ & $1(2)$ & $0(0)$ & $0(0)$ & .68 \\
Right temporal & $43(84)$ & $6(12)$ & $2(4)$ & $0(0)$ & .006 \\
Left frontal & $31(61)$ & $10(20)$ & $7(14)$ & $3(6)$ & $<.001$ \\
Left parietal & $37(73)$ & $5(10)$ & $7(14)$ & $2(4)$ & $<.001$ \\
Left occipital & $49(96)$ & $0(0)$ & $2(4)$ & $0(0)$ & .67 \\
Left temporal & $43(84)$ & $7(14)$ & $1(2)$ & $0(0)$ & $<.001$ \\
\hline
\end{tabular}

${ }^{a}$ Data are number of patients (\%).

Table 4: Global severity score and perinatal variables ${ }^{\mathrm{a}}$

\begin{tabular}{|c|c|c|c|c|c|}
\hline & $\begin{array}{c}\text { 1st Quartile } \\
(\text { Score }=1-5)(n=14)\end{array}$ & $\begin{array}{c}\text { 2nd Quartile } \\
\text { (Score }=6-11)(n=13)\end{array}$ & $\begin{array}{c}\text { 3rd Quartile } \\
\text { (Score }=12-25)(n=12)\end{array}$ & $\begin{array}{c}\text { 4th Quartile } \\
(\text { Score } \geq 26)(n=12)\end{array}$ & $P$ Value \\
\hline Gestation at birth (wk) & $38.2 \pm 1.3$ & $36.3 \pm 2.4$ & $36.7 \pm 2.5$ & $38 \pm 1.9$ & $.05^{\mathrm{d}}$ \\
\hline Birth weight (g) & $3445.8 \pm 618$ & $3066 \pm 989$ & $2947 \pm 619$ & $3247 \pm 570$ & $.34^{d}$ \\
\hline Male sex & $8(57)$ & $8(62)$ & $9(75)$ & $7(58)$ & $.87^{\mathrm{e}}$ \\
\hline 5-Minute Apgar & $7.8 \pm 1.6$ & $7.9 \pm 1.4$ & $8 \pm 1.5$ & $7.4 \pm 2.4$ & $.92^{\mathrm{d}}$ \\
\hline Compressions/epinephrine ${ }^{b}$ & $1(7)$ & $0(0)$ & $0(0)$ & $1(8)$ & $.97^{\mathrm{e}}$ \\
\hline Chorioamnionitis & $0(0)$ & $0(0)$ & $0(0)$ & $1(8)$ & $.98^{\mathrm{e}}$ \\
\hline Small for gestational age & $0(0)$ & $2(15)$ & $3(25)$ & 2 (17) & $.75^{\mathrm{e}}$ \\
\hline Neonatal infection ${ }^{c}$ & $1(7)$ & $2(15)$ & $3(25)$ & $3(25)$ & $.84^{\mathrm{e}}$ \\
\hline Ventilator requirement & $2(14)$ & $7(54)$ & $6(50)$ & $7(58)$ & $.18^{\mathrm{e}}$ \\
\hline Inotrope use & $0(0)$ & $3(23)$ & $2(17)$ & $4(33)$ & $.52^{\mathrm{e}}$ \\
\hline Seizures & $7(50)$ & $5(38)$ & $6(50)$ & $10(83)$ & $.25^{\mathrm{e}}$ \\
\hline
\end{tabular}

${ }^{a}$ Data are mean or number (\%).

${ }^{\mathrm{b}}$ Compressions or epinephrine or both were given in the delivery room during resuscitation.

cNeonatal infection is defined as systemic/serious infection in the first week of life.

${ }^{\mathrm{d}}$ The ANOVA was used to measure variance in means among 4 quartiles in continuous variables.

e The Kruskal-Wallis test was used for frequency distribution of categoric variables. be due a lack of MR imaging availability in most regional nurseries, necessitating transport to a neonatal intensive care unit with a higher level of care.

We compared regional WM injury scores with the global severity score and found that the occipital WM scores and WM cerebral edema scores were not significantly different among quartiles and therefore do not seem to impact the global severity score. Scores for all other regions were significantly different among quartiles. Thus, a modified, simpler score that excludes occipital and WM cerebral edema scores might be considered in toparietal area has been described previously., ${ }^{5,10}$ It has been hypothesized that the higher frequency of transcerebral veins in the temporal and occipital regions and the thinner WM and shorter medullary veins in the temporal region are protective factors and thus contribute to the predominantly frontoparietal pattern seen in our patients and others studied. ${ }^{5}$

We noted a difference in gestational age at birth among global severity score quartiles with infants of a slightly younger gestational age (36-37 weeks) clustered around the median in the second and third quartiles, and infants born at an older gestational age (38 weeks), in the first and fourth quartiles. This U-shaped distribution of gestational age and global severity score quartiles is likely not clinically significant but may suggest that other unidentified factors affect severity.

We observed no difference between the global severity score quartile and selected perinatal variables (Table 4 ). We considered using a neonatal disease-severity scoring system (such as the Scores for Neonatal Acute Physiology) to stratify our patients by clinical disease severity at the time of presentation. However, most scoring systems are based on physiologic variables or treatment in the first 24 hours of life. ${ }^{13}$ Most of our patients appeared healthy at birth and did not come to medical attention until postnatal days $4-5$, making use of these scoring systems inappropriate. future studies. This lesional distribution concentrated in the fron- 
Limitations of this study included the retrospective, nonharmonized imaging data. However, the scoring system is relatively simple and can be applied to routine MR neuroimaging sequences. We also acknowledge the sample size, which may have contributed to the lack of significance of some perinatal risk factors. However, this remains the largest cohort examined to date.

There is no method to predict prognosis for term and late preterm infants with perinatal DMV thrombosis and infarction. This standardized scoring system allows us to stratify patients by severity of objective radiologic findings at the time of diagnosis. Further investigation is needed to compare severity scores at diagnosis with outcome data. Correlation of long-term effects of lesions on motor, cognitive, and behavioral performance may lead to a predictive model to help guide parental counseling and recommend therapies and early intervention.

No clinical outcome data related to DMV pathology in neonates or children exist beyond a few isolated case reports. In another cohort, follow-up MR imaging studies demonstrated evolution of the lesions across time with WM necrosis, development of cystic spaces, volume reduction, and hyperintense signal intensity of the periventricular WM on T2-weighted and FLAIR images, yielding a pattern of appearance similar to that of periventricular leukomalacia. ${ }^{5}$ It is likely that for DMV pathology, as for periventricular leukomalacia, ${ }^{14,15}$ a scoring system could be used to evaluate the long-term association of perinatal lesions with motor, cognitive, and behavioral performance in prospectively acquired datasets. However, unlike the published periventricular leukomalacia scoring system, the current DMV thrombosis and infarction classification can only be used in late preterm or term infants because this insult is not related in pathophysiology to encephalopathy of prematurity.

\section{CONCLUSIONS}

A 102-point MR imaging scoring system provides a comprehensive and objective classification of WM injury after DMV thrombosis and infarction in late preterm and term neonates. The DMV WM injury global severity score is independent of gestational age and other antenatal risk factors. This standardized scoring system can be used to stratify patients into severity groups for future work on the neurodevelopmental outcomes of patients after DMV thrombosis and infarction.

Disclosures: Kristen L. Benninger-RELATED: Grant: 1R01HD081120-01A1 from the Eunice Kennedy Shriver National Institute of Child Health and Human Development, Comments: R01 to Nathalie L. Maitre.* *Money paid to the institution.

\section{REFERENCES}

1. deVeber G, Andrew M, Adams C, et al; Canadian Pediatric Ischemic Stroke Study Group. Cerebral sinovenous thrombosis in children. N Engl J Med 2001;345:417-23 CrossRef Medline

2. Moharir MD, Shroff M, Pontigon AM, et al. A prospective outcome study of neonatal cerebral sinovenous thrombosis. J Child Neurol 2011;26:1137-44 CrossRef Medline

3. Yang JY, Chan AK, Callen DJ, et al. Neonatal cerebral sinovenous thrombosis: sifting the evidence for a diagnostic plan and treatment strategy. Pediatrics 2010;126:e693-700 CrossRef

4. Saxonhouse MA. Thrombosis in the neonatal intensive care unit. Clin Perinatol 2015;42:651-73 CrossRef Medline

5. Arrigoni F, Parazzini C, Righini A, et al. Deep medullary vein involvement in neonates with brain damage: an MR imaging study. AJNR Am J Neuroradiol 2011;32:2030-36 CrossRef Medline

6. Taoka T, Fukusumi A, Miyasaka T, et al. Structure of the medullary veins of the cerebral hemisphere and related disorders. Radiographics 2017;37:281-97 CrossRef Medline

7. Friedman DP. Abnormalities of the deep medullary white matter veins: MR imaging findings. AJR Am J Roentgenol 1997;168:1103-08 CrossRef Medline

8. Okudera T, Huang YP, Fukusumi A, et al. Micro-angiographical studies of the medullary venous system of the cerebral hemisphere. Neuropathology 1999;19:93-111 CrossRef Medline

9. Vilan A, Ribeiro JM, Reis C, et al. Deep medullary veins and brain injury. J Pediatr 2018;200:290-90.el CrossRef Medline

10. Doneda C, Righini A, Parazzini C, et al. Prenatal MR imaging detection of deep medullary vein involvement in fetal brain damage. AJNR Am J Neuroradiol 2011;32:E146-49 CrossRef Medline

11. Konanki R, Varma DR, Ratha C, et al. Teaching NeuroImages: fetal deep medullary vein thrombosis presenting as progressive intracerebral hemorrhage. Neurology 2015;85:e5-6 CrossRef Medline

12. Harris PA, Taylor R, Thielke R, et al. Research Electronic Data Capture (REDCap): a metadata-driven methodology and workflow process for providing translational research informatics support. J Biomed Inform 2009;42:377-81 CrossRef Medline

13. Dorling JS, Field DJ, Manktelow B. Neonatal disease severity scoring systems. Arch Dis Child Fetal Neonatal Ed 2005;90:F11-16 CrossRef Medline

14. Martinez-Biarge M, Groenendaal F, Kersbergen KJ, et al. MRI based preterm white matter injury classification: the importance of sequential imaging in determining severity of injury. PLoS One 2016; 11:e0156245 CrossRef Medline

15. De Vries LS, Van Haastert IL, Rademaker KJ, et al. Ultrasound abnormalities preceding cerebral palsy in high-risk preterm infants. J Pediatr 2004;144:815-20 CrossRef Medline 